\title{
A New Technique of Treating Periodontal Disease- "Bone One Session Treatment (Bost)"
}

\author{
Navneet Kaur* \\ Department of Periodontics, Faculty of dentistry, Baba Farid University of Health sciences, India
}

*Corresponding author: Navneet Kaur, Department of Periodontics, Faculty of dentistry, National Dental College \& Hospital Derabassi Punjab, India.

Received Date: September 13, 2021

Published Date: October 04, 2021

\begin{abstract}
Periodontitis is a chronic multifactorial inflammatory disease associated with dysbiotic plaque biofilms and characterized by progressive destruction of the tooth-supporting apparatus. Its primary features include the loss of periodontal tissue support, manifested through clinical attachment loss (CAL) and radiographically assessed alveolar bone loss, presence of periodontal pocketing and gingival bleeding. Periodontitis is a major public health problem due to its high prevalence, as well as because it may lead to tooth loss and disability, negatively affect chewing function and aesthetics, be a source of social inequality, and impair quality of life. There are advancements at the level of diagnostic and treatment modality to arrest the periodontal infection through various modern techniques that are available today.

The primary strategy of periodontal treatment is control over the etiological factors along with microorganisms, resolution of inflammation and restoring the lost alveolar support. Traditional treatment therapy such as Surgical and non-surgical therapy are the major aspects of periodontal treatment. Discontended with the traditional therapy to defend oral microflora and repair the lost alveolar bone support may lead the clinicians and researchers to explore the new frontier and unique technique is Bone One Session Treatment (BOST). It is an aerobic treatment that eliminates the periodontal disease without an invasive procedure in the deepest pocket and supporting the alveolar bone. This review article gives a new direction, importance and evaluation significant pathway to treat the periodontal disease by Bone One Session Treatment technique.
\end{abstract}

Keywords: Non-invasive; Aerobic microflora; Periodontitis; BOST; Periodontal therapy; immune response

\section{Introduction}

Periodontal disease is a chronic inflammatory process characterized by bacterial etiology. It results in progressive, sitespecific destruction of the supporting tissues of the tooth, resulting in a typical pathological lesion [1]. The main aim of pocket therapy is to eliminate the microbial causative factors of periodontal disease. It is a well-established fact that periodontal diseases are caused by bacteria. Over time, this supra gingival plaque becomes more complex, leading to a succession of bacteria that are more pathogenic. Bacteria grow in an apical direction and become sub gingival, and eventually, as bone is destroyed, and periodontal pocket is formed. In periodontal pockets the bacteria form a highly structured and complex biofilm. As this process continues, the bacterial biofilm extends so far sub-gingivally that the patient cannot reach it during his regular oral hygiene regime. Bacteria within the biofilm are well protected from the host' s immunological mechanisms as well as from antibiotics used for treatment [2]. It is therefore logical to treat periodontal pockets by mechanical removal of local factors and also by disrupt ion of the sub gingival plaque biofilm itself.

Treatment of periodontal disease involves a fine balance of various non-surgical and surgical methods carried out in order to reduce periodontal pocket depth, access residual plaque, initiate the regeneration of periodontal supporting tissues and decrease the risk of disease progression. Subgingival antiinfective therapy performed together with self-performed plaque control provides significant benefits in clinical parameters and improvement of systemic inflammatory markers [3]. Nonsurgical periodontal treatment consists of professional removal of plaque and calculus, elimination of plaque retentive factors, oral hygiene instruction, chemical plaque control and antibiotic medication 
which have served as the gold standard of periodontal therapy for decades. Treatment of teeth with tooth/site-dependent factors is less predictable and bears an elevated risk of the progression of periodontal tissue breakdown [4]. Mechanical therapy alone may have limited effect on some periodontopathogens and fail to eliminate them in ecological niches [3].

Every periodontist's vision is practical and simple with an affordable way to treat periodontal disease while seems difficult to realize the vision, it is quite possible with the recent advanced techniques which are available in today's world. The main focus of periodontal treatment is to control the growth of periodontal pathogens and elimination of soft tissue inflammation and results in further restoration of lost alveolar support. Unsatisfied with traditional approaches to preserve the oral microflora and restore the lost alveolar support, resourceful clinician's, scientists and researchers have turned into unique and sometimes bizarre approaches and are exploring the new horizons as well as new directions in non-surgical periodontal treatment.

Dr William Hoisington has developed a newer technology as a treatment modality namely Tri Immunophasic Periodontal Therapy (TIP) and Bone One Session Treatment (BOST) which is a division of TIP therapy based on influencing the immune release related risk factors. BOST is a non-invasive microscopic aerobic treatment procedure that eradicate the periodontal pathogens which causes the periodontal diseases in the deepest pockets and supporting alveolar bone [5]. This therapy eradicates the aggressive anaerobic periodontal pathogens which not only causes the destruction of supporting periodontium but also creep to circulation which further leads to systemic complications such as osteoporosis, preterm low birth weight (PTLBW), infertility and coronary heart diseases.

\section{Objective and Rationale of Tri-Immuno-Phasic Therapy (TIP)}

The hypothesis behind this therapy is that the body is able to treat the periodontitis in the defensive phase system and promote the healing of lost periodontium in the same way as it occurs in repair and recovery phase. Under the right conditions, lost and damaged periodontal bone can just heal similar to the other skeletal bone in the human body. TIP periodontal therapy affects the local and individual factors that alter the progression of periodontal disease and improve healing, supporting the host body in all the three phases of immunity: Vigilant readiness, defense phase and repair and regeneration phase.

The goal is to treat and manage the periodontal diseases without the need of periodontal surgery. The primary aim and objective of this therapy is to promote the bone healing with a new healthy tissue. This new healthy tissue is filled into the periodontal pocket which are formed during the periodontal diseases.

A number of bacteria, viruses, yeasts and parasites play a role here; it is anaerobic periodontal pathogens with their virulence factors and intrusiveness that pave the way for synergies with other microorganisms to break the continuity of connective tissue and form pathological gingival and periodontal pockets. Through the opening of entrance to the deeper tissues and strata, bacteria and other microorganisms enter the circulation. Researchers and scientists in their studies found that periodontal pathogens are present and secrete the endotoxin or leukotoxins as a virulence factor in atherosclerotic plaques in the coronary arteries (Fiehn, et al. 2005). The ability of these periodontal pathogens to remotely destroy connective tissue is potentially alarming if it causes the artery to tear or accelerate the blockage. Healing of lost alveolar bone occur in a similar fashion as the healing of other bones in our body is the goal of aerobic periodontics.

\section{Various Steps Involved in BOST Procedure [6-8]}

1. Bacterial DNA testing

2. BOST treatment

3. Controlling biting forces

4. Aerobic oral hygiene regimen

5. Lifestyle, nutrition, and exercise.

\section{Bacterial DNA testing}

The primary focus in management of initiation of periodontal disease by determining the type and amount (number) of periodontal pathogens that are present as a resident in the gingival and periodontal pockets. With the help of bacterial DNA testing, you can determine the periodontal pathogens that causes the periodontal disease in a very précised manner. The early detection in DNA testing would also confirm and eliminates the unnecessary usage of antibiotics. However, some periodontal pathogens such as P. gingivalis, A. actinomycetemcomitans can not be able to controlled with just traditional treatments because of secretion of virulence factors like gingipains and leukotoxins.

The bacterial DNA testing is performed by inserting the absorbent paper points sub gingivally for approx. 15 seconds. Very well-developed periodontal pathogens in plaque or biofilm covers the tooth root at the level of attachment starts collecting on the absorbent paper point and samples are sent to the laboratory to find out the pathogens on the basis of DNA or RNA genetic material. This testing is fast, simple, comfortable, and painless procedure. It provides a scientifically accurate direction to find out which periodontal pathogens may initiate the periodontal disease. It also helps to select the precise antibiotic combination which may be bactericidal or bacteriostatic for the growth of microorganisms. Trials on the above aspects can give good therapeutic results which produces the resistant strains of microorganisms. Bacterial DNA testing is indicated and performed in patients who are suffering from periodontal diseases and before the orthodontic treatment. With braces, force will be put on the bone and oral hygiene will be more difficult. Aerobic hygiene is also important to prevent postorthodontic problems like gingival recession as well as gingivitis during treatment. 


\section{BOST Treatment}

A typical BOST procedure in a full mouth takes 4-5 hours in a single day. During the recovery period, this technique minimizes the damage to the gingiva, alveolar bone and periodontal apparatus. Basically, this technique uses the stretch flap technique that permit the access to the deepest areas of roots and bony surfaces without giving the incision [6]. This technique is totally non-invasive microsurgical procedure. It removes the main etiological factors like dental plaque and calculus along with periodontal pathogens which produced the endotoxins and virulence factors.

\section{Stretch Flap Technique [7]:}

1. The universal curette number $4 \mathrm{R}-4 \mathrm{~L}$ is inserted inside the periodontal pocket where the working end of the curette facing towards the surface of tooth and the inactive blunt end points towards the tissue to expose the sulcus. Light back pressure is applied to the tissue to initiate stretching while removing the plaque and calculus which is present sub gingivally (Figure 1).

2. Direction of a curette is changed to a circumferential manner starting at the angle to mobilize the tissue movement to prevent the tension on the papilla and create an incisional flap.

3. First, direct the curette head towards surface and run the instrument to remove adhered granulation tissue and obtain a smooth regular bony surface that helps to physically remove the periodontal pathogens and their virulence factors along with toxins sub gingivally into the periodontal pocket as well as from the porosities through fresh bleeding (Figure 2).

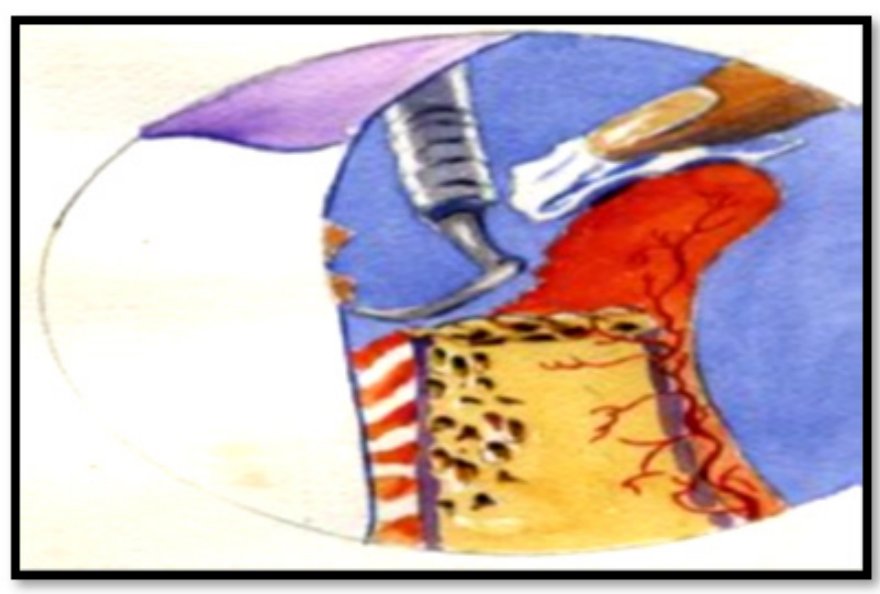

Figure 1: The gingiva is intentionally stretched using the rounded back of curettes [9].

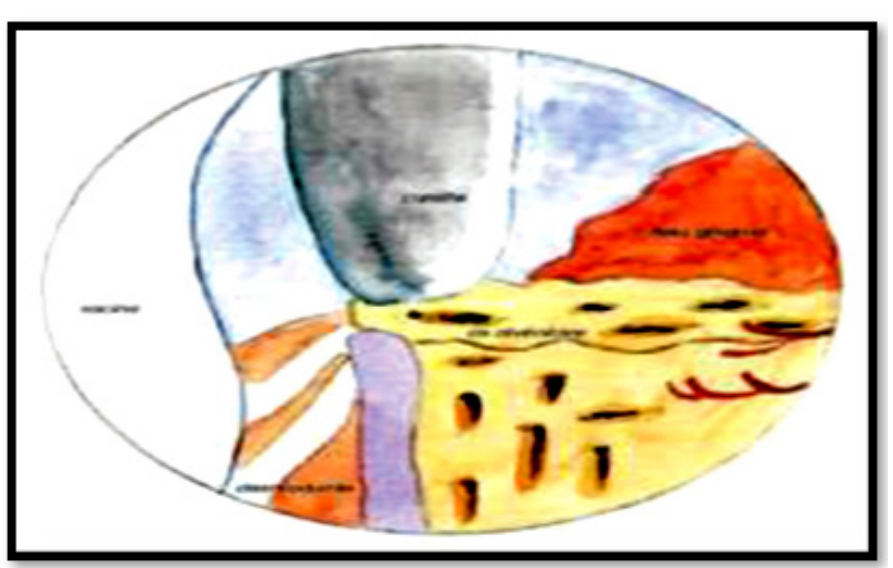

Figure 2: Cleaning and reshaping of bone craters.

This technique assures that the periodontium is left in an exceedingly state which will heal very effectively. By stretching the tissue, there are going to be no scar formation during the healing period. Scar formation always tries to prevent the attachment to tooth surface which implies that bone will stay weak after the traditional non-invasive periodontal treatment. It also permits the microorganisms to reinfect the tissue. However, stretch flap technique provide a powerful attachment to the tooth surface and healthy tissue starts filling the sub gingivally. 
BOST technique also have certain similarities with curettage however, it is different from the latter. Curettage procedure is used to remove the diseased granulation tissue and pocket lining without stretching the flap where as in this technique flap has been stretched slowly and remove the diseased granulation tissue to offer the stimulation of stem cells [7].

\section{Aerobic oral hygiene regimen}

\section{Control of occlusal forces and splinting}

Weakening of periodontium causes the normal occlusal forces to become traumatic. The treatment procedure includes the coronoplasty, enameloplasty and splinting to distribute the forces in an equilibrium. Control on occlusal forces prevent the tooth mobility and further tissue damage.

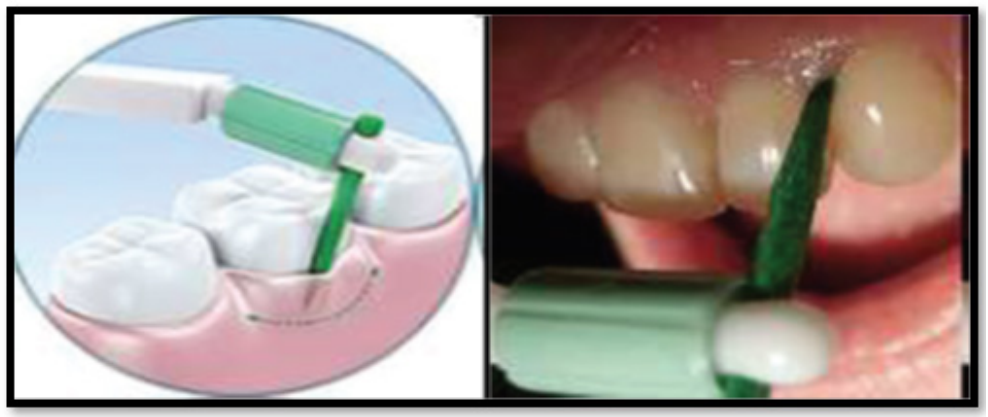

Figure 3: Perio-aid assisting in new attachment formation.

This step totally depends on the patient that how the patient maintains a daily oral hygiene regimen. Tooth brushing, dental floss interdental tooth brushes and other traditional methods of oral hygiene aids are used to maintain the oral hygiene. Aerobic oral hygiene kit (Perio-aid) (Figure 3) is used to clean the area sub gingivally and eradicate the disease causing anaerobic periodontal pathogens in deeper pockets, furcation and root concavities as these sites are far away to reach the dental floss or tooth brush. This Perio-aid stimulate the cells which are present at the base of the pocket (Figure 3).

\section{Lifestyle nutrition and exercise}

Eat balanced diet by eating regular meals, avoid of excessive sugar, avoid of excessive snacks, avoid granular food like nuts, seeds, chips, whole grain breads etc. Increase intake of vitamins and minerals, proteins. Vitamin c and zinc are most important. Avoid smoking, it reduces circulation and depresses certain immune cells. Regular exercise has to be done.

\section{Healing Phase After Bone One Session Treatment (BOST)}

After the completion of BOST procedure, healing should be preceded. The clot is firmly connected to the clean bone surface as a scaffold. The pocket is commonly very dense that is partially mineralized connective tissue and ultimately turns into acellular. The new mineralized acellular connective tissue (MAC) attachment will come to place in one month and the bone naturally heals. After bone healing there are 4 stages of therapy:

\section{Defensive phase: stem cell migrating phase: (Figure 4)}

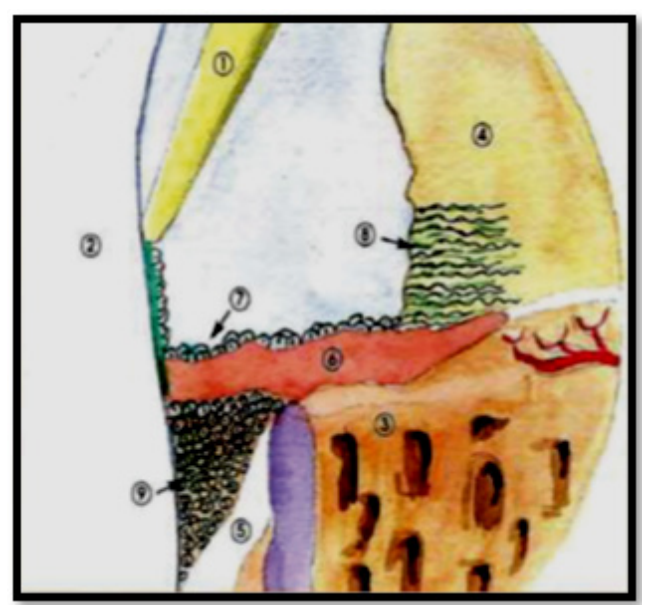

Figure 4: The clot that firmly attached to the clean bone serves as a scaffold. (1) Perio-aid, (2) tooth, (3) alveolar bone, (4) gingiva, (5) desmodont, (6) callus, (7 and 9) pluripotent stem cells, and (8) fibers. 
It includes the cleaning of bone aerobically to eliminate the periodontal pathogens as BOST immune reaction. This phase set up all the conditions where the host body can get out of the defensive phase against the periodontal pathogens and other microorganisms and enter a regeneration phase to heal back a new attachment. The stem cells inside the pocket moved along the root surface at the rate of $0.5 \mathrm{~mm} /$ day to thickened the layer of clot. A strict oral hygiene technique should be maintained that keeps the pocket open and keep the epithelial attachment away (Figure 4).

\section{Regeneration phase: Maturation of attachment: (Figure 5)}

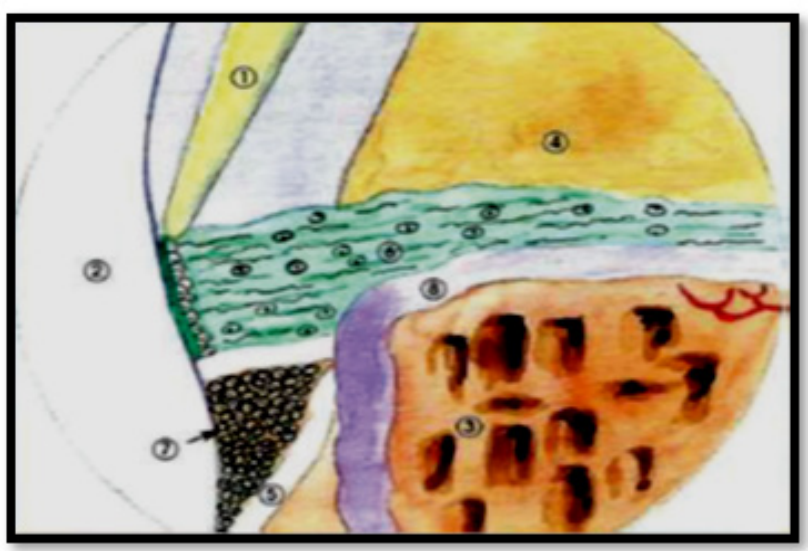

Figure 5: The pockets gradually fill in from the bottom with very dense, partially mineralized connective tissue. (1) Perio-aid, (2) tooth, (3) bone (4) gingiva, (5) desmodont, (6) mineralized acellular connective tissue, (7) pluripotent stem cells (8) New dense layer of osteoid formation.

As the healing progress on the root surface the pockets of 4-6 weeks and becomes acellular (Figure 5). gradually get filled with mineralized connective tissue for a period

\section{New attachment healing: (Figure 6)}

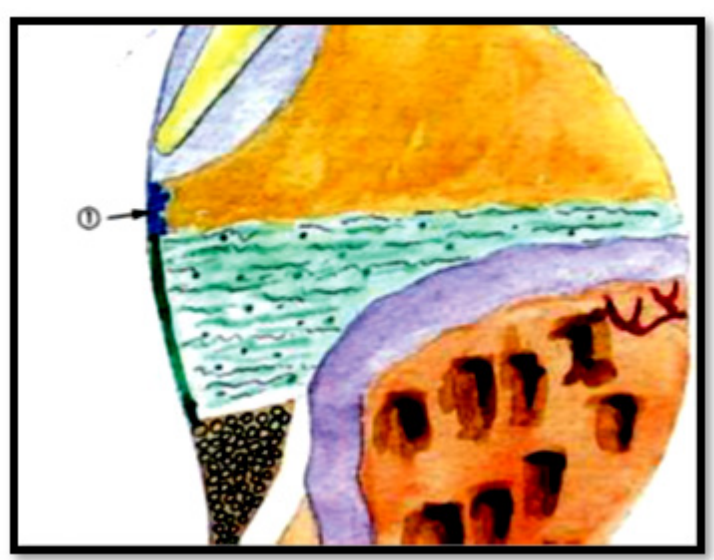

Figure 6: Mineralized acellular connective tissue attachment. (1) Zone of attachment.

Within the 1 month of the period after the formation of new mineralized MAC, bone naturally heals under it. A new dense layer of cortical bone forms over the healed inner cancellous bone in about 8 months (Figure 6).

4. The final healed result with the bone crest cortical layer reformed and the disappearance takes about nine months. This healing is visible on X-rays.

\section{Total Recovery Process}

DAY 1: BOST TREATMENT
DAY 2: PATIENT RESTART ORAL HYGINE REGIMEN

DAY 3: BLEEDING STOP AND CLOT STABILIZED

DAY 4: POCKETS START TO FILL IN

DAY 5: INFLAMMATION CONTINUES TO RECEED

DAY8: POCKET SEALING UP

DAY 14: RESUME NORMAL CHEWING REGIMEN

DAY 15: CHECK UP 
DAY 30: COMPLETE POCKET FILLED UP

DAY 35: ESTHETICS RESTORATION CAN BE STARTED.

DAY45: BONE IS BEGINNING TO HEAL. BY 9 MONTHS, BONE COMPLETELY HEALS

3 MONTHS: CHECK -UP

\section{Advantages of Bost}

1. Non-invasive and full-fledged aerobic therapy

2. Complete removal of granulation tissue from the pockets without incisions or sutures

3. Damage to underlying tissue is minimal

4. Quick reliable method and single session treatment

5. Healing is faster.

6. Relatively comfortable for a patient.

7. Less complications and no surgery is required.

8. No bone necrosis

9. Aesthetically more acceptable.

10. Less sensitivity.

\section{Conclusion}

As the progress keeps going in the field of technology and science, the advancements in order to identify the etiology of the disease and its treatment modalities will also keep going in dentistry. Clinicians should continue to develop newer advancements in diagnosis and treatment modalities as it is the main criteria to prevent progress of periodontal infection.

BOST technique introduced to conquer the disadvantages associated with the conventional treatment modalities in the periodontics. This technique gives a new direction to treat the periodontal disease in a non-invasive manner by changing the pathway of pathogenesis along with removal of etiological factors which further facilitates the regeneration repair and new attachment during the healing phase.

\section{Acknowledgment}

None.

\section{Conflict of Interest}

No conflict of interest.

\section{References}

1. Crispino A, Figliuzzi MM, Iovane C, DelGiudice T, Lomanno S, et al. (2015) Effectiveness of a diode laser in addition to non-surgical periodontal therapy: study of intervention. Annal Stomatologia(Roma) 6(1): $15-20$

2. Newman MG, Takei H, Klokkevold PR, Carranza FA (2011) Carranza's clinical periodontology. Elsevier health sciences.

3. Graziani, F, Karapetsa D, Alonso B, Herrera D (2000)(2017) Nonsurgical and surgical treatment of periodontitis: how many options for one disease? Periodontol 75: 152-188.

4. Tomasi C, Koutouzis T, Wennstrom JL (2008) Locally Delivered Doxycycline as an Adjunct to Mechanical Debridement at Retreatment of Periodontal Pockets. J Periodontol 79: 431-439.

5. William Hoisington (2006) New developments in perio: Tri-Immuno-Phasic therapy. Preventive Dentistry 1(2): 30-34.

6. Kiran KN, Chandhra MP, Ramesh B, Srikanth C, Arpita PR (2012) New trends in periodontics. J Evol Med Dent Sci 1: 546-548.

7. Kumar PY, Kalaivani V (2016) Tri-immuno phasic periodontal therapy. World J Pharm Res 5: 356-360.

8. Arpita R, Swetha JL, Babu MR, Sudhir R (2012) Recent trends in non-surgical periodontal care for the general dentist-A review. Bangla J Dent Res Educ 4: 78-82.

9. Parikh DH, Agrawal DC, Shah DK, Duseja DS, Shah DM, et al. (2019) Tri Immunophasic Periodontal Therapy (Tip) 2(1): 1-7. 